\title{
Coordination in hospitals: organized or emergent process?
}

\author{
Anne-Sophie Nyssen
}

Received: 1 October 2005/ Accepted: 1 October 2006

(C) Springer-Verlag London Limited 2006

\begin{abstract}
Hospitals have become more and more complex organizations that require increasing degrees of vertical, lateral and longitudinal coordination among their staff. As organizations, hospitals positively organize the coordination between the activities of its different agents, as well as the associated communication between them. The overall goal is to construct a "common ground" between the agents about the work process. In order to achieve this, a series of management tools are called upon. However these coordination mechanisms may fail. In the case analyzed, the agents seem to organize their behavior through direct and local individual interactions with their work environment, rather than through a global representation of the work. The case study demonstrates that intentionally organized coordination mechanisms interact with, and may be superseded by, the "emergencethrough use" of coordination mechanisms in real time. These two mechanisms are clearly embedded at work, and can both be beneficial in promoting coordination in large scale systems.
\end{abstract}

Keywords Organized coordination .

"Emergence-through-use" coordination · Health care . Accident analysis

A.-S. Nyssen $(\square)$

Cognitive Ergonomics, B32, FAPSE,

University of Liège, 4000 Liege, Belgium

e-mail: asnyssen@ulg.ac.be

\section{Introduction}

In recent years, hospitals have become more and more complex organizations that require increasing degrees of coordination and communication between health care providers. But, as investigations of medical accidents revealed (Chopra et al. 1992; Helmreich and Shaefer 1994), coordination systems occasionally fail, communication goes awry, errors occur resulting sometimes in dramatic consequences for the patient.

There have been two important developments in medical care relevant to the study of coordination in hospitals: (1) the specialization of medical sciences which has increased the division and distribution of tasks among experts from different disciplines and (2) the economical and political pressures that led hospitals to reorganize themselves into large scale networks in order to better allocate resources and costs.

Today, it is very seldom the case that a patient goes only to one hospital and sees only one physician during their stay. Multiple institutions, departments and professional skills are brought together in order to provide health services, but also to provide uninterrupted care around the clock. The fact that the patient's state itself changes and evolves over time makes it necessary for the diagnosticians to continuously update the data from the various available sources, and deal with evolution and histories.

In view of these properties, most medical situations require information to be exchanged between the individuals that work cooperatively in hospitals in order to coordinate interventions both in time and space. This coordination, to quote Savoyant (1977), covers two movements: integration of the actions distributed among the different areas of expertise, and temporal 
integration of actions and synchronization. In a previous article (Nyssen and Javaux 1996), we studied the problem of temporal coordination and synchronization constraints from an individual perspective and show, how anesthetists, to synchronize their behaviors with the surgeon's activity, transform complex duration problems into simple sequential problems using temporal references derived from their direct and regular interactions with the work environment. Errors occur when these temporal references are lacking. This paper deals more particularly with the modalities of coordination in hospitals at a group level. It does not attempt to provide a systematic review of the literature on this theme, but rather tries to briefly present the coordination requirements in hospitals, and to match, within the use of different frameworks, how the hospital assumes that coordination is achieved, against how people actually coordinate their activities.

\section{Coordination requirements}

One can describe three sets of coordination requirements in hospitals: vertical, lateral and longitudinal coordination needs.

\subsection{Vertical coordination}

A hospital, as any organization, structures its work and activities through a vertical distribution of power and decision making responsibilities. This is particularly reinforced in teaching hospitals where there are attendants or specialists at the top of the structure that can include a division, a clinic or a ward, and students at the bottom of the hierarchy. Because of their various positions in the hierarchy, individuals are likely to have differential access to information and knowledge; Exchange of information is required to keep the subordinates' supervisor aware of the situation and decisions. A vertical flow of information is needed, both bottom up and top-down, to provide the right people with the right information.

\subsection{Lateral coordination}

Another important aspect of a hospital is the existence of different domains of expertise that are institutionalized and organized into different units, with different technologies and specific subcultures. In the collective activity, each expert acquires a partial cognitive representation of the patient. The diagnosis and treatment of the patient require the coordination of activities across these different expertise domains and units and, in many cases, the evaluation and integration of the information from these various areas, including laboratory reports and medical histories goes into one global base of knowledge, if not a shared representation.

\subsection{Longitudinal coordination}

Medical activities themselves are made up of subsets of activities or sequences of actions that must be executed in the proper form and with the appropriate timing. These process constraints shape the coordination of work either at the longitudinal or the lateral coordination levels. Furthermore, the medical diagnosis process is a complex and incremental task; patients' information is permanently subject to modification as the result of physicians' actions, as well as the result of a spontaneous evolution of the patient's state; The diagnostician must continuously perceive and receive information from the various people and/or technological equipment that monitor the patient and update his/her representation of the patient.

\section{The hospital approach to coordination: centralized and external representations of work processes}

The hospital itself, as any organization, positively organizes the coordination between the activities of its different agents, as well as the communication between them. The overall goal here seems to be to construct "common ground" between the agents regarding the process that turns the activities into cooperative work and provides some shared knowledge about the situation. In order to achieve this, it calls on a series of conventional management tools that specify ad hoc patterns of behavior, and shape directly or indirectly the interactions and the communication between the agents. Examples of such tools are hierarchy, work organization process, patient process, procedures and instructions, and the like.

This coordination approach refers to the Common Knowledge Theory (Lewis 1969; Krauss and Fussell 1990) and is derived from the classical assumption that the success of coordination lies in the extent to which the community and individual agents are prepared to understand and share "common ground". In work studies, the idea of "common ground" shared by the individuals who perform a collective activity led to the concepts of "functional referential "(Leplat 1991), or "mental model" (Norman 1987) that define work processes and allow group members to organize their activities. 
Such conventional tools exist for each coordination requirement:

\subsection{Vertical coordination tools}

The work organization in a university hospital allocates the simplest part of the collaborative-work patient task to the novices and the more complex part to the more experienced workers. Novices' work is commonly monitored by residents and/or seniors. In many hospitals, a phone communication network is organized in a cascade to provide, at any time, help from those who are more experienced. The basic functioning of vertical coordination is that the novices do their work, with some internal and external "sentry markers" (events, parameters, ..) which tell them that it is time to call for assistance from someone at a higher level of expertise. The key issue is therefore the relevance of these "sentry markers" that alert the novices and suggest a call at the right time. In a previous study (De Keyser and Nyssen 1993), we showed that trainees often fail to estimate correctly how long they should reasonably wait before calling, and do so too late: either they overestimate their competences, or they underestimate the speed of the patient's deterioration, or sometimes they do not want to lose face.

\subsection{Lateral coordination tools}

The resources of the hospital (either technical or human) are both specialized and limited and a careful coordination of the activities, both in time and in space across the facilities, is required. This resource management is based on multiple planning activities, which are organized according to different time frames: they may start up to a year beforehand, and then evolve from annual, to monthly, to daily and hourly schedules. Computerized systems are used to exchange information between people from different areas in order to gather the right people at the right time and place and achieve lateral coordination.

The work process itself also defines how the tasks are organized among the agents and shape their interactions. Individual and collective patterns and sequences of behavior are defined into procedures.

\subsection{Longitudinal coordination tools}

There are rotations of multiple teams in charge of the patient around the clock in a hospital. A transition period is generally planned in the agent's schedule to allow for data transmission briefings.
One important tool for longitudinal (and lateral) coordination is the patient's records, either in its paper form or in its computerized form. For both the hospital and for the team, it is a means to trace and memorize the state of the patient, and the actions of the different agents around the clock. It contains the history of the case, contextual information, the distributed dynamic diagnosis and the treatment process. Each agent is supposed to fill in the patient's records with his/her contribution to the actions and information and, so, to transmit his/her knowledge to the next agent. By accumulating information over time and from different agents, the patient's records play a critical role in coordinating. It is intended to produce the global representation of the patient's situation to enable an isolated agent to solve dynamic problem situations.

The hospital normally takes for granted that the staff will adhere to these coordination principles and, so doing, assumes that coordination problems are solved. Communication is obviously the key issue behind these conventional tools. However, in the following case description, I will show how these coordination mechanisms may fail. As French ergonomists would like to put it, there is a difference between the coordination mechanisms as expected and organized by the hospital, and the coordination process that can be actually observed in the field in real time.

\section{Case study}

One night on a weekend, a 16-year-old patient showed up at the reception desk of the emergency room of hospital A for respiratory distress related to a problem of chronic asthma. The clinical examination was done by a resident who prescribed treatment (inhaled bronchodilator and antibiotic therapy) and let the patient go home.

Later in the night, his respiratory distress symptoms reappeared at a higher degree. In the early morning, the patient showed up at the emergency room of a larger hospital (B) in the same area. The patient was in an agitated and anxious state. He was directed to a room of unit $\mathrm{X}$ where he was examined by resident 1 (R1) and monitored (ECG, arterial blood. pulse, ...). The clinical examination did not show any acute respiratory problems. $\mathrm{R} 1$ gave the patient some treatment and kept him for close monitoring. At 9AM (at the end of his shift), R1 transmitted all the information concerning all the patients to the resident 2 (R2). In the middle of the morning, the patient felt better and $\mathrm{R} 2$, after examination, decided to let the patient go. 
In the early afternoon, the patient showed up again at the emergency department of hospital B, still complaining about respiratory distress. At the reception desk, the secretary recognized the patient. For her, there was no high emergency and, this time, she decided to refer the patient to Unit Z. Unit $Z$ had just been created in the emergency department in order to take care of minor emergencies. General practitioners (GP) from outside the hospital were used in that unit for patient care. In that unit, the patient was examined by a GP who was on call in the hospital for her fifth time. The nurse, who was working with the GP, also recognized the patient and informed her about the case. The nurse went to get the patient's records and, at the same time, asked R2 to come and see the patient.

The GP and R2 were both in the room and examined the patient. The patient showed some signs of tachycardia, nasal flaring, hypoxemia and anxiety. R2 decided to give the patient oxygen and started corticoids and GP proposed to give him some anxiolytics. In the afternoon, the patient complained about chest pains. The GP added some analgesic to the treatment and called a psychologist who didn't diagnose any particular mental problems.

At the end of the afternoon, a nurse came to see the GP to tell her that the patient was not complaining anymore and seemed better. She asked her to come and examine the patient in order to see if he could go home. After close examination, she decided to let the patient go. R2 heard later that the patient had left.

In the evening, the patient went into respiratory arrest at home. He is taken by ambulance to hospital B but too late.

\section{Case analysis: an emergence-through-use approach of coordination}

Through the course of the event, it is not easy to identify where and how the case goes wrong. This difficulty can be faced for many accident analyses that involve multiple actors and actions distributed in time and space. There is no one error or one factor easily identifiable at the origin of the case. Each decision, taken separately, is relevant in its finite temporal interval. The failure comes from the lack of the temporal integration of the decisions and actions distributed in time and between the agents. I have developed above how coordination in hospitals is assumed to be achieved through the use of centralized coordination principles and tools. In the situation presented above, this centralized organization of coordination failed to achieve the process of integration of knowledge necessary for handling the complex problem. Confronted to an unusual situation, the agents organized their behavior through direct and local interactions with the work environment, based on their understanding of the situation. However, this local process of coordination disorganized the anticipated sequence of operations. Let us analyze in detail the coordination modes and failures.

\subsection{At the hospital network level}

Hospitals A and B are two units in the same urban area. For a potential patient, they are two equivalent solutions, two interchangeable structures, in other words two units of the same hospital network. Hence there is no issue in going to hospital A first, then to hospital B. But this implies some connections between the two units that are lacking in the example. Each unit creates its own patient record. In the case study, there was no communication of any type (verbal or written) between the emergency units of the two hospitals. However, it must be noted that this lack of communication could have been overcome by the application of a recent national measure that designates the patient as the agent who keeps his exams record files.

\subsection{At the emergency department-level}

There is a procedure written by the chief of the department that organizes the patient orientation across the different sub-units of the emergency department, and so lateral coordination. However, this algorithm was not used by the agents in the case described above, and is actually not used by the agents. They are not really informed about it, and they were not involved in its development process. Furthermore, the algorithm does not cover the case of a patient who comes back again several times in the same day. Actually, the first line agent, the receptionist, achieves coordination without formal procedure. He/she identifies the degree of emergency, the nature of the problem and attempts to match the demands with the available resources. This matching is mediated by interactions with colleagues, and supported by a computerized system that gives an external representation of the work load of the different sub-units. This global representation of the team's activities is permanently updated, yet does not keep track of past activities. Its goal is to help the management of resources. It was not intended to help the agent for longitudinal coordination. 


\subsection{At the team-level}

The coordination between GP and R2 is something that emerges from a set of local interactions rather than by the implementation of the procedure that explicitly organizes the transmission of information between physicians. In the case study, it was the nurse who detected the presence of the patient the second time, and organized the transmission of information through the patient's records and by direct verbal interaction between R2 and GP. Doing this, the nurse created a system in which everyone believed that the others knew everything about the task, hence shared the same understanding in a fully cooperative work. This emergent movement of increasing expertise results in a pattern of overlapping expertise rather than of cooperating work. In fact, the knowledge of the medical task is represented most redundantly, but the centralized knowledge necessary for a dynamic problem solving is paradoxically represented least redundantly, and is lost across the agents as well as vertical and lateral coordination.

\subsection{At the physician dyad-level}

Each physician used the patient's records but everything happened as if each agent started a new reasoning process instead of integrating the information recorded in the patient's records and constructing a global dynamic representation. Clearly, the individual performance of the agents was not improved by the use of this external memory source assumed to achieve longitudinal coordination. Even the direct verbal interaction among individuals did not really help to alert the physicians and provide any diagnostic benefit. In contrast, these local interaction and local coordination processes might actually have worked against the efficiency of the problem solving process by confusing each person's role and creating some kind of "stammering" in the reasoning and treatment process.

This failure to build a cooperative work process can be related to two cognitive processes: (1) the fact that the patient's records constitute a "source memory" that can unconsciously favor "plagiarism" in the problem solving process. This phenomenon has been largely studied in social psychology and is called cryptomnesia. The result is a fixation behavior that, in case of diagnosis error, propagates the reasoning failure between the agents. In other circumstances, the same behavior can create a kind of longitudinal convergence that can actually improve group performance; (2) the fact that the patient's records constitute an "external" memory containing events that the readers have not necessarily experienced themselves. In a kind of egocentric behavior, each agent seems to re-start the diagnosis game repeatedly instead of continuing the game with different players every time. A positive aspect of this is that, by replaying the game every time, agents create redundancy; each agent can detect someone else's error and update the patient's representation with new information and current interactions with the environment. But, there is no real mechanism for developing the cross-individual learning as would be necessary to construct a historical and global representation of the patient's situation.

\section{Discussion}

Coordination problems in large scale hospital systems are problems of interdependent and dynamic decision making in which multiple experts must deal with the history and evolution of the patient's state. Up to now, hospitals have dealt with the coordination problems by developing centralized tools such as procedures, work processes, instructions, that specify the work and the activities across time and distance and guide interactions. Hospitals normally take for granted that the staff will adhere to these coordination principles and, so doing, assume that coordination problems are solved. The above situation shows how these centralized coordination mechanisms may fail, either because they are not adhered to by individual agents, or because the critical information for coordination is not memorized or not transmitted; Reasons for these failures can be, for instance, that the agents aren't familiar with the conventional tool, that the tool does not cover a particular case, that the computerized system is not designed for all the coordination needs: vertical, lateral and longitudinal.

Another basic assumption of the hospital for successful coordination relies upon the establishment of patients' records that track the patient's situation over time, and provide, by accumulation, the knowledge necessary for an isolated agent to solve complex and dynamic problem situations. This knowledge is important because it provides agents with all the background information. However, we have shown that the use of the patient's records is not a guarantee for successful coordination. We have demonstrated that each agent, behaving egocentrically, seems to give precedence to his/her own current perception of the situation based on his/her direct and real time interactions with the patient, and re-starts the reasoning process instead of continuing it. This kind of behavior is non-optimal in our situation. However, as mentioned 
above, it creates a kind of redundancy that might sometimes improve the performance of the system in some circumstances of diagnostic error.

Obviously, instead of centralized coordination mechanisms, the case analysis clearly demonstrates the "emergence-through use" of coordination in real time. Each agent seems to organize the activities through direct and local interactions in his/her work environment.

Recent research (Bonabeau and Theraulaz 1994; Gilbert and Conte 1995) that studies, by analogy, coordination in insects and non human societies shows local coordination mechanisms at the origin of very complex patterns of animal societies. For instance, Reynolds (1987) demonstrated that the flocking behavior of birds can be simulated by assuming that individual birds make local adjustments based on the velocity and bearing of neighboring birds. Thus, despite appearances, such complex flocking behavior is generated by local coordination processes rather than by global centralized ones.

The issue here is not to argue in favor or against one of the two approaches of coordination described above: "centralized and external coordination tools" approach versus "local and emergence-through-use" approach. Our case study is remarkable, in part for the way in which it shows both the richness and the fragility of a partial coordination approach either organized at the blunt end or developed at the sharp end. Contrary to what a pure "blunt end" perspective about human and social performance sometimes assume or pretend, an external and centralized organization of coordination cannot handle the variability of complex dynamic systems at all relevant levels of details. The efficiency of coordination in such systems seems also depends critically on local, auto-organized coordination processes. But, contrary to some naive assumptions about sharp end performance, local regulations are not always virtuous; they can disorganize the sequence of operations, as designed through the centralized process. Indeed, local regulations themselves depend on the agents' capacity to "make sense" of the patient's situation, and therefore, ironically, to develop and maintain an integrated representation of the situation-particularly to keep track of patients' medical histories through (and despite) the current, local and direct interactions of individual agents with their work environment. Such an integrated representation of the situation seems necessary for individual agents to palliate some natural cognitive biases, aggregate the information distributed in time and across the agents, and properly adjust their responses.

It is evident that the two approaches of coordination are clearly embedded at work, and can both be beneficial in promoting coordination, but may also be counteracting each other if not mutually recognized. Particularly, the conditions for facilitating, or obstructing, individual integrated representations may well be dominantly determined by organizational design features. The important avenue for future research is in fact to understand how these approaches actually interact, and might be combined to improve safety in large scale systems.

\section{References}

Bonabeau E, Theraulaz G (1994) Intelligence collective. Hermès, Paris

Chopra V, Bovill JG, Spierdijk J, Koorneef F (1992) Reported significant observations during anaesthesia: a prospective analysis over 18-month period. Br J Anaesth 68:13-17

Helmreich R, Schaefer H-G (1994) Team performance in the operating room. In: Bogner MS (ed) Human error in medicine. Lawrence Erlbaum Associates, Hillsdale, NJ, pp 225-273

De Keyser V, Nyssen AS (1993) L'erreur humaine en anesthésie. trav hum 56:243-266

Gilbert N, Conte R (1995) Artificial societies: the computer simulation of social life. Ucl Press, London

Krauss RM, Fussel SR (1990) Mutual knowledge and communicative effectiveness. In: Galegher $\mathbf{J}$ (ed) Intellectual teamwork: social and technological foundations of cooperative work. Lawrence Erlbaum Associates, Hillsdale, NJ, pp 111-145

Leplat J (1991) Organization of activity in collective tasks. In: Rasmussen J (ed) Decision making: cognitive models for cooperative work. Wiley, New York, pp 51-71

Lewis D (1969) Convention: a philosophical study. Harvard University Press, Cambridge, MA

Norman DA (1987) The psychology of everyday things. Basic Books, New York

Nyssen AS, Javaux D (1996) Analysis of synchronization constraints and associated errors in collective work environments. Ergonomics 39:1249-1264

Reynolds CW (1987) Flocks, herds, and schools: a distributed behavioral models. Comput Graph 21:25-34

Savoyant A (1977) Coordination et communication dans une équipe de travail. trav hum 40(1):41-54 